\title{
Análisis segmentario del pelo para detectar la exposición crónica a drogas psicoactivas
}

\section{Segmental hair testing to disclose chronic exposure to psychoactive drugs}

\author{
Emilia Marchei*, Ilaria Palmi*, Simona Pichini*, Roberta Pacifici*, Ileana-Rita Anton Airaldi*, \\ Juan Antonio Costa Orvay**, Joan García Serra**, Bartolomé Bonet Serra**, Oscar García- \\ AlgAR ${ }^{* * *}$
}

"Unidad de Abuso de Drogas y de Adicciones, Departamento de Investigación Terapéutica y Evaluación de Medicina, Instituto Superior de Sanidad, Roma, Italia; ${ }^{* *}$ Servicio de Pediatría, Hospital Can Misses, Ibiza, España; ${ }^{* * *}$ Grupo de Investigación Infancia y Entorno (GRIE), Servicio de Pediatría, Instituto Hospital del Mar de Investigaciones Médicas (IMIM), Barcelona, España.

\section{Resumen}

Se presenta el caso de un niño sano de 4 años de edad que ingresa en la sala de hospitalización pediátrica por la sospecha de una intoxicación accidental debido a la ingesta de fármacos narcolépticos (metilfenidato, sertralina y quetiapina), que tomaba de forma pautada su hermano de 8 años de edad que padecía un síndrome de Asperger. La evaluación objetiva de la intoxicación se puede realizar con la determinación de los fármacos y sus metabolitos en matrices biológicas con una ventana de tiempo corta (sangre y orina) o larga (pelo).

En el hospital se realizó un análisis de sangre y orina mediante inmunoanálisis (confirmado mediante espectrometría líquidacromatografía de masas) y se identificó la presencia de sertralina y quetiapina y sus metabolitos. Con la sospecha de administración crónica de fármacos al niño, se procedió al análisis del pelo con cromatografía líquida de ultra-alto rendimiento-espectrometría de masas en tándem. El pelo se dividió en 6 segmentos consecutivos de $2 \mathrm{~cm}$ de longitud, de forma que permitieron estudiar la ingesta de los fármacos durante los últimos 12 meses. En los primeros 4 segmentos se encontró quetiapina con una concentración media de $1,00 \mathrm{ng} / \mathrm{mg} \pm 0,94 \mathrm{ng} / \mathrm{mg}$ de pelo y en todos los segmentos se encontraron sertralina y su metabolito, desmetil-sertralina, con una concentración media de 2,65 \pm 0,94 ng/ mg y $1,50 \pm 0,94 \mathrm{ng} / \mathrm{mg}$ de pelo, respectivamente. El análisis de pelo resultó negativo para metilfenidato y su metabolito (ácido ritalínico).

La detección en matrices biológicas de fármacos psicoactivos demostró la intoxicación aguda y crónica por quetiapina y sertralina, administradas por la madre.

Palabras clave: Análisis segmentario del pelo; Niños; Antidepresivos; Antipsicóticos; Cromatografía líquida de ultra-alto rendimientoespectrometría de masas en tándem.

\section{Abstract}

This study presents the case of a 4-year-old healthy child admitted to the paediatric ward for suspected accidental intoxication due to ingestion of narcoleptic drugs (methylphenidate, sertraline and quetiapine), taken on a regular basis by his 8-year-old brother affected by Asperger syndrome.

Intoxication can be objectively assessed by measurements of drugs and metabolites in biological matrices with short-term (blood and urine) or long-term (hair) detection windows.

At the hospital, the child's blood and urine were analysed by immunoassay (confirmed by liquid chromatography-mass spectrometry), and sertraline and quetiapine and their metabolites were identified. The suspicion that the mother administered drugs chronically prompted the analysis of six, consecutive 2-cm segments of the child's hair, using ultra-high performance liquid chromatographytandem mass spectrometry, thereby accounting for ingestion over the previous 12 months. Quetiapine was found in the first four segments with a mean concentration of $1.00 \mathrm{ng} / \mathrm{mg} \pm 0.94 \mathrm{ng} / \mathrm{mg}$ hair while sertraline and its metabolite, desmethyl-sertraline, were found in all segments with a mean concentration of $2.65 \pm 0.94 \mathrm{ng} / \mathrm{mg}$ and $1.50 \pm 0.94 \mathrm{ng} / \mathrm{mg}$ hair, respectively. Hair analyses were negative for methylphenidate and its metabolite (ritalinic acid).

Biological matrices testing for psychoactive drugs disclosed both acute and chronic intoxication with quetiapine and sertraline administered by the mother

Keywords: Segmental hair testing; Children; Antidepressants; Antipsychotics; Ultra-high performance liquid chromatographytandem mass spectrometry. 
$\mathrm{D}$ ada la severidad de los trastornos pediátricos del estado de ánimo y de ansiedad, y sus consecuencias psicosociales y funcionales, la práctica clínica requiere el desarrollo de enfoques complementarios para casos complejos, por ejemplo, la combinación de antidepresivos con otras drogas psicotrópicas (Díaz-Caneja, Espliego, Parellada, Arango y Moreno, 2014). La polifarmacia psiquiátrica pediátrica, definido como la ingesta diaria de varios medicamentos psiquiátricos para el mismo o varias condiciones, ha aumentado (Hilt et al., 2014). Los antidepresivos se han convertido en una de las clases de fármacos recetadas en mayor frecuencia en combinación, y es habitual su prescripción conjunta con estimulantes y antipsicóticos en niños y adolescentes afectados por trastornos psiquiátricos (Díaz-Caneja et al., 2014). La preocupación sobre esto incluye la insuficiente evidencia de estudios sobre la efectividad de gran parte de las combinaciones de los medicamentos, y la pobre definición de los efectos secundarios de las combinaciones de medicamentos (Hilt et al., 2014). En particular, los efectos secundarios fueron más comunes en las combinaciones de medicamentos que incluían inhibidores selectivos de la recaptación de serotonina (ISRS) o antipsicóticos (Hilt et al., 2014).

Algunos casos han revelado reticencia, pero también el consumo particular o excesivo, más una administración criminal forzada por parte de los padres, principalmente en combinación, y esto potencialmente puede resultar en efectos secundarios severos (Binchy, Molyneux y Manning, 1994; Gaillard et al., 2011; Pawłowicz, Wasilewska, Olanski y Stefanowicz, 2013).

La evaluación objetiva de la intoxicación se puede realizar mediante la detección de los fármacos y sus metabolitos en matrices biológicas con una ventana de tiempo corta (sangre y orina) o larga (pelo); en particular, el análisis de pelo ha mostrado éxito en cuanto a intoxicaciones crónicas pediátricas (García-Algar et al., 2005; Joya et al., 2009, 2011; Papaseit, García-Algar, Simo, Pichini y Farre, 2013; Pichini et al., 2006, 2014a, 2014b, 2014c).

Además, el análisis segmentario del pelo puede revelar las eventuales exposiciones crónicas mes-a-mes (usando segmentos de pelo de $1 \mathrm{~cm}$ ) y, en algunos casos, identificar patrones de consumo/administración de drogas (Pichini et al., 2006; Thieme, Baumer, Sachs y Teske, 2013).

Detallamos el caso de un niño admitido al hospital por la sospecha de intoxicación con drogas psicoactivas y cuyo análisis segmentario del pelo reveló una intoxicación crónica.

\section{Método}

\section{Estudio de caso}

El servicio pediátrico del hospital ingresa a un niño de 4 años que tiene dolor en sus extremidades inferiores y torpeza. El día previo a su ingreso, la familia notó que su somnolencia aumentaba y que su marcha sufría alteracio- nes. Durante el examen inicial en urgencias el niño estaba agotado, tenía somnolencia, carecía de fiebre, tosía y tenía secreción nasal espesa. El niño fue ingresado con el diagnóstico de encefalitis versus intoxicación de drogas (dos años antes había sido ingresado debido a un episodio de encefalitis que fue resuelto en un periodo de tres meses). Los resultados fueron normales en cuanto al recuento de células rojas, las pruebas del funcionamiento del hígado y del riñón, incluyendo un análisis del líquido cerebroespinal para determinar la presencia de lactatos y de amonio, tanto como de un TAC de la cabeza. La cromatografía líquida de ultra-alto rendimiento-espectrometría de masas de sangre y orina reveló la presencia de sertralina y de quetiapina, dos de los tres fármacos psicoactivos (el tercero era metilfenidato) que tomaba el hermano de 8 años, afectado por el síndrome de Asperger.

Una vez hospitalizado, el niño presentó un amplio espectro de síntomas, tales como somnolencia persistente, debilidad generalizada, lenguaje ininteligible, incontinencia urinaria y estreñimiento. Durante los primeros cuatro días, experimentó miosis, lagrimeo y blefaroespasmo. Los síntomas clínicos cambiaron gradualmente durante cuatro días: ningún reflejo profundo a partir del segundo día, una erupción eritematosa en el tercio superior del tórax, cabeza y cuello, movimientos distónicos de las extremidades, señales extrapiramidales y convulsiones tónicas generalizadas con midriasis escasamente reactivo a la luz, tratado con diazepam. A raíz de esto, fue ingresado en la unidad de cuidados intensivos (UCI) para intensificar su tratamiento y monitoreo. Pocas horas después de ser transferido a la UCI, el niño experimentó una recuperación gradual de su estado de alerta y su tono muscular, finalizó su midriasis, pudo sentarse por sí mismo, sujetó objetos y los movió entre las manos y los alzó. Se observó mejora de su lenguaje, incluyendo la comprensión de órdenes sencillas. Después de 24 horas, el niño fue capaz de andar.

La severidad del cuadro clínico, la similitud del episodio anterior, y la presencia de ambas drogas en las muestras de sangre y de orina motivaron la solicitud del análisis segmentario del pelo, dada la sospecha fundamentada de la administración crónica y no accidental por parte de la madre de metilfenidato, sertralina y quetiapina, que también se administraban a su hermano que había sido prescrito dicho tratamiento.

\section{Recogida, preparación y análisis de la muestra}

Pelo del niño, con una medida de $12 \mathrm{~cm}$, fue cortado en 6 segmentos de $2 \mathrm{~cm}$, representando una ventana de tiempo de aproximadamente 2 meses por segmento, para un total de 12 meses. Dichas muestras de pelo fueron analizadas para la presencia de metilfenidato, sertralina y quetiapina y sus metabolitos (ácido ritalínico y desmetil-sertralina) y cualquier otro abuso de droga eventual. En el momento del análisis, el metabolito de quetiapina 7-hidroxi-quetiapina 
no estaba disponible. Resumiendo brevemente, las muestras de pelo (20 mg) se cortaron en trazos más cortos y, después de su descontaminación con diclorometano y metanol, se añadió $10 \mu \mathrm{l}$ usando como patrón interno (prometazina 2 $\mu \mathrm{g} / \mathrm{ml}$ ) y tratado con $500 \mathrm{ml}$ M3 de solución amortiguadora (Comedical, Trento, Italia) durante una hora a $100{ }^{\circ} \mathrm{C}$. Después, las muestras tratadas se enfriaron a temperatura ambiente y $100 \mu \mathrm{L}$ del extracto M3 fue diluido con $900 \mu$ $\mathrm{L}$ de agua antes de analizar un volumen de muestra de 10 $\mu \mathrm{L}$ mediante cromatografía líquida de ultra-alto rendimiento-espectrometría de masas en tándem. La cromatografía de fase reversa se realizó usando una columna de Acquity UPLC HSS C18 $(2.1 \mathrm{~mm} \times 150 \mathrm{~mm}, 1.8 \mu \mathrm{m})$ con una elución en gradiente lineal con dos disolventes: $0,1 \%$ ácido fórmico en acetonitrilo (disolvente A) y $0,1 \%$ ácido fórmico en agua (disolvente B). El disolvente A se mantuvo al $10 \%$ durante el primer 0,5 minuto. Se incrementó hasta el $55 \%$ entre minutos 0,5 a 4 , se mantuvo al $55 \%$ entre minutos 4 a 6 , y después se volvió a disminuir hasta el $10 \%$ entre minutos 6 a 6,1 min y se mantuvo al $10 \%$ entre minutos 6,1 a 10 para su re-equilibrado. La velocidad de flujo se mantuvo constan- te, a 0,4 $\mathrm{mL} / \mathrm{min}$ durante el análisis. Los analitos separados se detectaron con un espectrómetro de masas triple cuadrupolo triple operado en modo monitorización de reacciones múltiples (MRM) usando ionización por electrospray (ESI) positivo. Las condiciones ESI fueron las siguientes: voltaje capilar de $3.0 \mathrm{kV}$, temperatura de evaporación del solvente de $600{ }^{\circ} \mathrm{C}$, temperatura de la fuente de $150{ }^{\circ} \mathrm{C}$, velocidad de flujo del gas del cono de $60 \mathrm{~L} / \mathrm{h}$, velocidad de flujo de evaporación del solvente de $1100 \mathrm{~L} / \mathrm{h}$ y velocidad de flujo de gas de colisión de $0.13 \mathrm{~mL} / \mathrm{min}$. Se definieron los valores para los voltajes de la energía del cono, las transiciones MRM, y los voltajes de la energía de colisión para cada analito (Tabla 1). El método fue validado según descripciones previas (Pichini et al., 2014a) y aplicado con un límite de cuantificación de $0,1 \mathrm{ng} / \mathrm{g}$ y un límite de detección de 0,04 $\mathrm{ng} / \mathrm{g}$. La linealidad varió entre $0,1 \mathrm{a} 10 \mathrm{ng} / \mathrm{g}$. La imprecisión fue menor del $10 \%$, la recuperación analítica varió entre el $70,1 \%$ y el $95,3 \%$ y la eficiencia del proceso fue del $80,9 \%$. Todos los analitos estudiados mostraron supresión/ aumento de iones no significativos (menos de $10 \%$ de supresión de la señal analítica debido al efecto de la matriz).

Tabla 1. Cromatografía líquida de ultra-alto rendimiento-espectrometría de masas en tándem en modo de monitorización de reacciones múltiples (MRM)

\begin{tabular}{|c|c|c|c|c|c|c|c|}
\hline \multirow[t]{3}{*}{ Analitos } & \multirow[t]{3}{*}{ Tiempo de retención (min) } & \multicolumn{6}{|c|}{ Transiciones MRM } \\
\hline & & \multicolumn{3}{|c|}{ Cuantificación } & \multicolumn{3}{|c|}{ Confirmación } \\
\hline & & $\mathrm{m} / \mathbf{z}$ & CV (V) & CE $(\mathrm{eV})$ & $\mathrm{m} / \mathbf{z}$ & $C V(V)$ & CE $(\mathrm{eV})$ \\
\hline Ácido Ritalínico & 2,99 & $220,3>84,2$ & 30 & 16 & $220,3>174,2$ & 30 & 20 \\
\hline Metilfenidato & 3,51 & $234,3>84,1$ & 26 & 18 & $234,3>56,1$ & 26 & 32 \\
\hline Quetiapina & 4,16 & $384,2>221,2$ & 26 & 36 & $384,2>253,2$ & 26 & 20 \\
\hline Desmtil-sertralina & 5,48 & $292,2>159,0$ & 8 & 28 & $292,2>275,1$ & 8 & 16 \\
\hline Sertralina & 5,68 & $306,2>159,1$ & 14 & 30 & $306,2>275,1$ & 14 & 14 \\
\hline Prometazina (IS) & 4,84 & $285,2>198,1$ & 22 & 28 & & & \\
\hline
\end{tabular}

\section{Resultados}

Tabla 2 muestra los resultados de los análisis del pelo usando los seis segmentos de $2.0 \mathrm{~cm}$ recogidos después de la admisión hospitalaria del niño. No había presencia de metilfenidato y su metabolito, ácido ritalínico, en ningún segmento analizado, mientras que sí que había presencia de quetiapina en todos los segmentos correspondientes a los últimos ocho meses, y de sertralina y su metabolito desmetil-sertralina en todos los segmentos correspondientes a los últimos doce meses.

Tabla 2. Drogas psicoactivas en la muestra de pelo del niño intoxicado

\begin{tabular}{|c|c|c|c|c|c|}
\hline Segmento $(\mathrm{cm})$ & Metilfenidato (ng/mg) & Ácido Ritalínico (ng/mg) & Quetiapina (ng/mg) & Sertralina (ng/mg) & Desmetil-sertralina (ng/mg) \\
\hline $0-2$ & nd & nd & 2,29 & 4,28 & 3,79 \\
\hline $2-4$ & nd & nd & 1,23 & 3,28 & 1,89 \\
\hline $4-6$ & nd & nd & 0,40 & 2,07 & 0,87 \\
\hline $6-8$ & nd & nd & 0,10 & 2,25 & 1,66 \\
\hline $8-10$ & nd & nd & nd & 1,88 & 0,41 \\
\hline $10-12$ & nd & nd & nd & 2,13 & 0,39 \\
\hline
\end{tabular}


La concentración de quetiapina disminuye desde 2.29 $\mathrm{ng} / \mathrm{mg}$ en el segmento proximal hasta $0.10 \mathrm{ng} / \mathrm{mg}$ en el segmento distal.

Se halló sertralina y su metabolito, desmetil-sertralina, en todos los segmentos. No obstante, la concentración de sertralina fue bastante estable en los segmentos distales y aumentaba únicamente en los segmentos correspondientes a los últimos cuatro meses, mientras que la concentración de los metabolitos disminuía del segmento proximal al distal.

\section{Discusión}

El análisis del pelo de un niño de cuatro años hospitalizado con síntomas neurológicas de una posible intoxicación mostró una intoxicación crónica con dos drogas psicoactivas: quetiapina y sertralina, debido a la administración criminal por parte de su madre de las drogas prescritas a su hermano.

Son pocos los datos publicados sobre la concentración de quetiapina y sertralina en pelo. Únicamente encontramos un estudio publicado que informaba sobre la concentración de quetiapina en el pelo de adultos tratados con dosis de entre 200 y 1200 mg (Binz, Yegles, Schneider, Neels y Crunelle, 2014). Las concentraciones de quetiapina variaron entre 0,35 a $10,21 \mathrm{ng} / \mathrm{mg}$ pelo, con concentraciones de 7-hydroxy-quetiapina de entre 0,02 y 3,19 ng/mg pelo en los segmentos de pelo con una longitud de dos cm o más. Los individuos mostraron una tendencia en cuanto a la concentración de quetiapina similar a la nuestra: un descenso lineal del segmento proximal al distal. La hipótesis de los autores es que es más probable que dicha disminución de la concentración se deba al efecto de tratamientos capilares cosméticos (p. ej., champús y otros productos) que a un cambio en la reticencia o las dosis. Esto es coherente con el efecto reconocido internacionalmente de tratamientos capilares cosméticos y del efecto del sudor en la disminución de la concentración xenobiótica desde los segmentos proximales hasta los distales (Jurado, Kintz, Menendez y Repetto, 1997). De manera similar, sólo un estudio informó de la concentración de sertralina en pelo humano post-mortem con concentraciones de sertralina de entre 0,6 y $1,6 \mathrm{ng} /$ mg pelo, con variaciones de las concentraciones de desmetil-sertralina de entre 0,5 y $2.6 \mathrm{ng} / \mathrm{mg}$ pelo, de una persona fallecida (Wille et al., 2009).

Aunque son escasos los datos de análisis de pelo de quetiapina y sertralina en niños, podemos concluir, dados los resultados obtenidos, que el niño fue tratado con ambas drogas de manera continua. En general, las manifestaciones clínicas de la toxicidad de las drogas antipsicóticas incluye varios grados de depresión del sistema nervioso central, efectos anticolinérgicos, cambios pupilares, convulsiones, hipotensión, y anormalidades de la conducción cardiaca (Cobaugh et al., 2007). Es más, muchos pacientes que sufren una sobredosis con inhibidores selectivos de la recapta- ción son asintomáticos (Sarko, 2000). Los síntomas, cuando se manifiestan, suelen ser autolimitados y consisten de taquicardia, somnolencia, temblores, náuseas, agitación, alucinaciones visuales, diaforesis, eritema y vomiteras (Grenha, Garrido, Brito, Oliveira y Santos, 2013; Myers, Dean y Krenzelok, 1994; Pao y Tipnis, 1997).

En nuestro estudio de caso, los síntomas del niño que resultaron de la ingestión de drogas psicotrópicas y la exposición crónica fueron establecidos en base a la presencia de quetiapina y sertralina en el pelo. El pico de la concentración de la droga ocurre en la sección de pelo de 0 a 2 $\mathrm{cm}$ para ambas quetiapina y sertralina, sugiriendo que estas drogas fueron ingeridas de manera accidental, intencional o forzada, en cuantías no insignificantes antes de la intoxicación aguda y, como consecuencia, la hospitalización.

Las implicaciones clínicas principales de este caso se relacionan con la utilidad del análisis de pelo para descubrir el consumo o la exposición crónicos a drogas recetadas (y también en el caso de drogas adictivas) en relación a sobredosis, combinación o polifarmacia.

\section{Conclusiones}

El análisis de pelo es complementario a las analíticas de sangre y de orina para descubrir una intoxicación crónica sospechada en relación a xenobióticos tóxicos en la presencia y/o ausencia de una intoxicación aguda. Además, el análisis segmentario del pelo puede aportar información acerca de si la sustancia se ingirió de manera continua con anterioridad del supuesto incidente, o si la sustancia se ingirió únicamente durante un periodo corto que corresponde al momento del incidente.

\section{Reconocimientos}

Los autores desean expresar su gratitud a Dra. Claudia Mortali, Dra. Luisa Mastrobattista y Dra. Adele Minutillo por su asistencia técnica y a Recercaixa por su soporte.

\section{Conflicto de intereses}

Los autores declaran la inexistencia de conflictos de intereses.

\section{Referencias}

Binchy, J. M., Molyneux, E. M. y Manning, J. (1994). Accidental ingestion of methadone by children in Merseyside. British Medical Journal, 308, 1335.

Binz, T. M., Yegles, M., Schneider, S., Neels, H. y Crunelle, C. L. (2014). Time resolved analysis of quetiapine and 7-OH-quetiapine in hair using LC/MS-MS. Forensic Science International, 242, 200-203. doi: 10.1016/j.forsciint.2014.07.002 
Cobaugh, D. J., Erdman, A. R., Booze, L. L., Scharman, E. J., Christianson, G., Manoguerra, A. S. y Troutman, W.G. (2007). Atypical antipsychotic medication poisoning: an evidence-based consensus guideline for out-of-hospital management. Clinical Toxicology, 45, 918-942.

Díaz-Caneja, C. M., Espliego, A., Parellada, M., Arango, C. y Moreno, C. (2014). Polypharmacy with antidepressants in children and adolescents. International Journal of Neuropsychopharmacology, 17, 1063-1082. doi: 10.1017/ S1461145712001265

Gaillard, Y., Breuil, R., Doche, C., Romeuf, L., Lemeur, C., Prevosto, J. M. y Fanton, L. (2011). Detection of amitriptyline, nortriptyline and bromazepam in liver, CSF and hair in the homicidal poisoning of a one-month-old girl autopsied 8 months after death. Forensic Sciences International, 207, e16-e18. doi: 10.1016/j.forsciint.2010.11.010

Garcia-Algar, O., López, N., Bonet, M., Pellegrini, M., Marchei, E. y Pichini S. (2005). 3,4-methylenedioxymethamphetamine (MDMA) intoxication in an infant chronically exposed to cocaine. Therapeutic Drug Monitoring, 27, 409-411.

Grenha, J., Garrido, A., Brito, H., Oliveira, M. J. y Santos, F. (2013). Serotonin syndrome after sertraline overdose in a child: a case report. Case Reports in Pediatrics, 2013: 897902. doi: 10.1155/2013/897902.

Hilt, R. J., Chaudhari, M., Bell, J. F., Wolf, C., Koprowicz, K. y King, B. H. (2014). Side effects from use of one or more psychiatric medications in a population-based sample of children and adolescents. Journal of Child and Adolescent Psychopharmacology, 24, 83-89. doi: 10.1089/ cap.2013.0036

Joya, X., Papaseit, E., Civit, E., Pellegrini, M., Vall, O., Garcia-Algar, O.,... Pichini, S. (2009). Unsuspected exposure to cocaine in preschool children from a Mediterranean city detected by hair analysis. Therapeutic Drug Monitoring, 31, 391-395. doi: 10.1097/FTD. 0b013e31819c3f2b

Joya, X., Fríguls, B., Simó, M., Civit, E., de la Torre, R., Palomeque, A.,... Garcia-Algar, O. (2011). Acute heroin intoxication in a baby chronically exposed to cocaine and heroin: a case report. Journal of Medical Case Reports, 5, 288. doi: 10.1186/1752-1947-5-288. doi: 10.1186/1752$1947-5-288$

Jurado, C., Kintz, P., Menéndez, M. y Repetto, M. (1997). Influence of the cosmetic treatment of hair on drug testing. International Journal of Legal Medicine 110, 159-163.

Myers, L. B., Dean, B. S. y Krenzelok, E. P. (1994). Sertraline (Zoloft): A) pediatric overdose approach. Veterinary and Human Toxicology, 36, 361.

Pao, M. y Tipnis, T. (1997). Serotonin syndrome after sertraline overdose in a 5 year old girl. Archives of pediatrics and adolescent medicine, 151, 1064-1067.

Papaseit, E., García-Algar, O., Simó, S., Pichini, S. y Farré, M. (2013). Methylphenidate in the treatment of chil- dren with attention-deficit hyperactivity disorder: monitoring in biological matrices. Anales de Pediatria, 78, 123. e1-e123.10. doi: 10.1016/j.anpedi.2012.08.015

Pawłowicz, U., Wasilewska, A., Olanski, W. y Stefanowicz M. (2013). Epidemiological study of acute poisoning in children: a 5-year retrospective study in the Paediatric University Hospital in Białystok, Poland. Emergency Medicine Journal, 30, 712-716. doi: 10.1136/emermed-2012-201376

Pichini, S., Poudevida, S., Pujadas, M., Menoyo, E., Pacifici, R., Farré, M. y de la Torre, R. (2006). Assessment of chronic exposure to MDMA in a group of consumers by segmental hair analysis. Therapeutic Drug Monitoring, 28, 106-109.

Pichini, S., Gottardi, M., Marchei, E., Svaizer, F., Pellegrini, M., Rotolo, M. y Pacifici, R. (2014a). Rapid extraction, identification and quantification of drugs of abuse in hair by immunoassay and ultra-performance liquid chromatography tandem mass spectrometry. Clinical Chemistry and Laboratory Medicine, 52, 679-686. doi: 10.1515/cclm-2013-0784

Pichini, S., Rotolo, M. C, García, J., Girona, N., Leal, L., García-Algar, O. y Pacifici, R. (2014b). Neonatal withdrawal syndrome after chronic maternal consumption of 4-methylethcathinone. Forensic Science International, 245C, e33-e35. doi: 10.1016/j.forsciint.2014.10.027

Pichini, S., Garcia-Algar, O., Alvarez, A., Gottardi, M., Marchei, E., Svaizer, F.,... Pacifici, R. (2014c). Assessment of unsuspected exposure to drugs of abuse in children from a Mediterranean city by hair testing. International Journal of Environmental Research and Public Health, 11, 2288-2298. doi: 10.3390/ijerph110202288

Sarko, J. (2000). Antidepressants, old and new. A review of their adverse effects and toxicity in overdose. Pharmacologic Advances in Emergency Medicine, 18, 637-654.

Thieme, D., Baumer, C., Sachs, H. y Teske, J. (2013). Screening and long term retrospection of psychoactive drugs in presumptive drug-facilitated crimes using segmented single hair. Drug Test Analysis, 5, 736-740. doi: 10.1002/ dta. 1502

Wille, S.M., De Letter, E.A., Piette, M.H., Van Overschelde, L.K., Van Peteghem, C.H. y Lambert, W.E. (2009). Determination of antidepressants in human postmortem blood, brain tissue, and hair using gas chromatography-mass spectrometry. International Journal of Legal Medicine, 123: 451-458. doi: 10.1007/s00414-008-0287-6. 